a pathway to identify potential candidates for surgery. Their clinic letters were reviewed to determine whether the surgical option had been considered. A literature review was undertaken to identify key factors needed to develop a referral framework.

Results Among 381 patients identified, 19 patients were excluded having insufficient data and 30 having undergone previous lung resection. In the remaining 322 patients (Figure 1), 89 patients had localised disease, were fit, with adequate respiratory reserve. In this group, we further identified 8 patients ( $2 \%$ of study population) who were potential candidates for surgery. None of these patients had been considered for surgery. Following a discussion at the Midland Bronchiectasis Network meeting with input from experienced thoracic surgeons, a referral framework was generated to assist physicians in identifying potential candidates.

Conclusions We identified 8 patients who could benefit from surgery. Although they constitute a small proportion of the study population $(2 \%)$, the result suggests respiratory physicians may be under-referring patients for surgery. A referral framework is introduced to assist physicians in referring appropriate patients to a surgical team.

\section{Clinical Characterisation of Idiopathic Pulmonary Fibrosis}

\section{P272 EPIDEMIOLOGY OF IDIOPATHIC PULMONARY FIBROSIS IN THE UK: FINDINGS FROM THE BRITISH LUNG FOUNDATION'S 'RESPIRATORY HEALTH OF THE NATION' PROJECT}

${ }^{1} \mathrm{~N}$ Snell, ${ }^{2} \mathrm{D}$ Strachan, ${ }^{3} \mathrm{R}$ Hubbard, ${ }^{3} \mathrm{~J}$ Gibson, ${ }^{4} \mathrm{~T}$ Maher, ${ }^{1}$ I Jarrold. ${ }^{1}$ British Lung Foundation, London, UK; ${ }^{2}$ St George's, University of London, London, UK; ${ }^{3}$ University of Nottingham, Nottingham, UK; ${ }^{4}$ Royal Brompton Hospital, London, UK

10.1136/thoraxjnl-2016-209333.415

Introduction and objectives The last comprehensive survey of UK respiratory disease epidemiology was the British Thoracic Society's 2006 Burden of Lung Disease report. We performed an analysis covering 2004-2012. Findings pertaining to IPF are presented here.

Methods Prevalence and incidence rates were estimated from a primary care database (the Health Intelligence Network) representing $\sim 5$ per cent of the population, using a broad range of primary care codes considered to encompass the IPF definition. Mortality figures were derived from official government statistics. For international mortality comparisons and numbers of hospital admissions/inpatient bed-days we used WHO data.

Results An estimated 32,500 people in the UK live with IPF, a prevalence rate of about 50/100,000. This is more than double NICE's 2015 estimate of 15-25 per 100,000. There are around 6,000 new cases diagnosed/year, greater than previous estimates of around 5,000. Overall, 5,300 people/year die from IPF, slightly more than the previous commonly accepted estimate of 5,000. There are nearly 9,000 admissions/year for IPF, accounting for around $1.3 \%$ of all admissions due to lung disease and $1.4 \%$ of all hospital bed days, despite IPF affecting less than $0.25 \%$ of people who have had a lung disease diagnosis. IPF is $50 \%$ more common in men, and killed $60 \%$ more men than women from 2008-2012. In this period 13,974 men and 8,624 women died from IPF, broadly in line with previous estimates. Incidence increases with age, around $85 \%$ of diagnoses being made in people aged over 70. Prevalence is highest in Northern Ireland, north-west England, Scotland and Wales. IPF is least common in London. Incidence is not influenced by measures of deprivation.

Conclusions Although rare, IPF is considerably more common than previously recognised and represents a small but significant burden on NHS hospital services.

\section{P273 BASELINE CHARACTERISTICS OF PATIENTS WITH IDIOPATHIC PULMONARY FIBROSIS AGED OVER 80 YEARS OLD}

${ }^{1}$ GA Margaritopoulos, ${ }^{2} \mathrm{~A}$ Proklou, ${ }^{3} \mathrm{D}$ Badenes Bonet, ' $\mathrm{M}$ Kokosi, 'TM Maher, 'EA Renzoni, ${ }^{1} \mathrm{AU}$ Wells, ${ }^{1} \mathrm{~F}$ Chua. 'Interstitial Lung Disease Unit, Royal Brompton Hospital, London, UK; ${ }^{2}$ Thoracic Medicine Department, University Hospital of Herakleion, Herakleion, Crete, Greece; ${ }^{3}$ Pneumology Department, Hospital del Mar, Barcelona, Spain

\subsection{6/thoraxjnl-2016-209333.416}

Introduction and objectives Idiopathic Pulmonary Fibrosis (IPF) is strongly associated with advanced age. Our aim is to identify if the baseline characteristics of patients aged $>80$ who are excluded from pharmaceutical trials differ compared to patients aged $<80$ years.

Methods Consecutively diagnosed IPF patients $\geq 80 \quad(n=61)$ and $<80(\mathrm{n}=320)$ presenting to the ILD Unit of the Royal Brompton Hospital from 1/1/2010 to $31 / 12 / 2013$ were included in this analysis. Data regarding age, smoking status, pulmonary function tests including $\left(\mathrm{FEV}_{1}, \mathrm{FVC}, \mathrm{DLco}\right.$ and $\left.\mathrm{Kco}\right)$ were extracted from medical records. All cases were discussed by an ILD multi-disciplinary panel for the purpose of assigning a consensus diagnosis.

Results The baseline characteristics of both study groups are summarised in Table 1. Patients were also grouped by smoking status; the smoking history of 10 patients in the $\geq 80$ and 3 patients in the $<80$ groups was unavailable.

We divided both groups to smokers and non-smokers. FEV 1 and FVC did not differ between non-smokers from both study groups whereas were both significantly lower in the smokers aged $<80$ years than in smokers aged $>80$ years $(74,44 \pm 18,24$ versus $86,34 \pm 12,67 \mathrm{p}=0.0005$ for $\mathrm{FEV}_{1}$ and 72,96 $\pm 20,77$ versus 88,34 $\pm 13,68 \mathrm{p}=0.0001$ for FVC). DLco and Kco did not differ between smokers and non-smokers.

Conclusion Patients with IPF aged $\geq 80$ years present with higher spirometric values than those $<80$ in particular when a history of smoking is taken into consideration. Mean FVC did not differ between the non-smoking cohorts in each age-separated group while baseline DLco and Kco were not different between the two study groups regardless of smoking status. The higher FVC in smokers with IPF aged $>80$ compared to younger smokers could be due to the concomitant presence of radiologically evident emphysema. This finding has clinical implications with respect to eligibility for NICE-approved anti-fibrotic treatment. Analysis of the extent of emphysema in these patients and further studies of a higher number of patients is underway. 